REVESCO. Revista de Estudios Cooperativos

ISSN: $1885-8031$

https://dx.doi.org/10.5209/REVE.67338

\title{
Las plataformas colaborativas como oportunidad para la innovación social
}

\author{
Juan Manuel Dieste Cobo' ${ }^{1}$
}

Recibido: 28 de junio de 2019 / Aceptado: 1 de abril de 2020 / Publicado: 13 de abril de 2020

Resumen. Teniendo como punto de contacto las plataformas digitales, la interacción entre la economía colaborativa y la economía social podría generar beneficios mutuos, explotando sinergias que favorezcan su mutua consolidación. La evolución de la economía colaborativa le ha situado en un espacio intermedio entre la economía tradicional, de la que pretende ser un modelo alternativo, y formas de economía social o cooperativa. Cuando las plataformas "colaborativas" solo están diseñadas para reducir costes y maximizar beneficios se habla de "capitalismo de plataforma", un modelo que, aunque aparentemente similar, resulta en esencia opuesto al "cooperativismo de plataforma", otra forma de economía colaborativa que combina lo mejor de las plataformas digitales con los principios cooperativistas internacionales. La transformación de un modelo a otro es consecuencia de favorecer la propiedad compartida, la puesta en marcha de nuevos modelos de gobernanza, la participación del ciudadano productor de valor en la toma de decisiones en términos de igualdad y el reparto de los beneficios entre los usuarios. De esta forma el valor añadido del cooperativismo de plataforma es generado por todos los agentes que interactúan a través de la plataforma digital, es decir, tanto los proveedores como los usuarios, por lo que se desarrolla un proceso de cocreación interactiva y los principios y valores cooperativos promueven el empoderamiento de los prousers. Por tanto, estos valores pueden impulsar la transformación de las organizaciones desde el modelo corporativo al cooperativo, pasando por el colaborativo, lo que supondría no solo un profundo cambio económico, sino todo un cambio de paradigma sociocultural.

Palabras clave: Economía social; Economía colaborativa; Capitalismo de plataforma; Cooperativismo de plataforma; Plataformas digitales; Innovación social.

Claves Econlit: A13; L31; M14; P13.

\section{[en] Collaborative platforms as an opportunity to reach social innovation}

\begin{abstract}
Social and sharing economy have some issues in common, like, sometimes, digital platforms. Both models have also a great complementary potential to create synergies and pursue its mutual strengthening. Perhaps sharing economy is faced with a major challenge in the coming years: to move towards a platform cooperativism model, in which digital platforms reflect the principles and values of cooperativism, or instead of, to be absorbed by the corporate economy, more interested in reduce costs and increase benefits through this platforms. Differences between these options are related to how the resources and goods are shared, the governance model adopted and the involvement of empowered citizens who are conscious of their role as producers, and not only as users or consumers. Therefore, the application of the cooperative values can contribute, not only to improved business performance and to reach cooperative governance, but also to make progress towards a new social and cultural paradigm, focused on promoting human and social development.
\end{abstract}

Keywords: Social economy; Sharing economy; Corporate sharing economy; Platform cooperativism; Digital platforms; Social innovation.

Sumario. 1. Introducción. 2. Economía social y economía colaborativa. 3. Las plataformas digitales. 4. Cooperativismo o capitalismo de plataforma. 5. Conclusiones. 6. Referencias bibliográficas.

Cómo citar: Dieste Cobo, J.M. (2020) Las plataformas colaborativas como oportunidad para la innovación social. REVESCO. Revista de Estudios Cooperativos, vol. 133, e67338. https://dx.doi.org/10.5209/REVE.67338.

\section{Introducción}

Las innovaciones tecnológicas han tenido y tendrán en el futuro un impacto que va mucho más allá de las personas, empresas o instituciones que las promueven o utilizan. Recientemente se cumplieron 30 años de la creación de Internet, momento que marcó el inicio de la era digital, un profundo cambio aún en curso, comparable con el que, en su tiempo, significó la revolución industrial. El desarrollo exponencial de estas

\footnotetext{
Universidad de La Laguna, España

Dirección de correo electrónico: jdiestec@ull.edu.es.
} 
nuevas tecnologías, y su confluencia con los avances de otros campos, nos sitúan ya en la cuarta revolución industrial, donde conceptos como el internet de las cosas, hasta hace poco inimaginables, están cada vez más presentes. Su adopción como herramienta cotidiana a escala social ha supuesto la transformación del paradigma comunicativo y el modo de interacción, propiciando un cambio en el modelo de relaciones interpersonales, pasando de un carácter netamente individualista a un nuevo patrón más abierto y grupal.

A nivel global, las nuevas tecnologías están reconfigurando la forma en que se hacen negocios, se estudia y se busca entretenimiento. Tapscott y Williams (2010) se refieren a este fenómeno como la "wikinomía", la creciente accesibilidad de las tecnologías de la información que permiten a cualquiera colaborar, crear valor y competir en la innovación y la creación de riqueza en todo sector de la economía. Es decir, la nueva web 2.0 permite la creación de comunidades que comparten conocimientos y otros recursos y servicios que cualquiera puede utilizar o modificar, dando lugar a un nuevo mundo donde se crean y se consumen colaborativamente noticias, descubrimientos y todo tipo de contenidos.

De esta forma se construyen ecosistemas sociales propios, donde se cohesionan comunidades en torno a objetivos sociales concretos, como son el diseño y la construcción de soluciones efectivas, frente a necesidades sociales detectadas y no atendidas suficientemente por la Administración. Se observa así un cambio de paradigma que ha trascendido desde lo meramente tecnológico y comunicativo, hasta alcanzar el campo de lo social y participativo. (Sajardo y Pérez, 2019)

Es en este marco donde surgen las plataformas colaborativas, que suponen un modelo de negocio emergente dentro de lo que inicialmente se denominó "consumo colaborativo" y, a consecuencia de su desarrollo exponencial, en los últimos años "economía colaborativa". A pesar de que su conceptualización es todavía un tanto difusa, sí hay consenso en apuntar algunos elementos característicos de la economía colaborativa, como la función de los agentes que interactúan a través de las plataformas digitales, estableciendo relaciones de colaboración o intercambio comercial, al margen de las tradicionales estructuras empresariales. Esta capacidad, a su vez, explica el potencial de este modelo para favorecer el emprendimiento empresarial, su especial capacidad de adaptación a los cambios del entorno y su rápida extensión a diversos sectores económicos.

Algunas de estas características no son extrañas a las que definen a la economía social. Teniendo su raíz en el asociacionismo, la relevancia de estas entidades se debe actualmente a la necesidad de transformar un modelo económico que ha provocado exclusión social, un grave daño medioambiental y finalmente una crisis económica global sin precedentes.

Sin embargo, uno de los retos para el crecimiento y la propia supervivencia de estas formas de economía social y colaborativa es el uso eficiente de estas nuevas tecnologías, especialmente las plataformas digitales; para la consecución de sus fines, adaptándolas a sus principios y valores. En ello radica a nuestro juicio la verdadera esencia de estas entidades y su capacidad de resiliencia, en poner por encima de criterios empresariales una visión humanista, social y eco-responsable. En consecuencia, el objetivo de este trabajo es valorar el potencial de las plataformas digitales en la economía social y colaborativa, como herramienta en la necesaria apuesta por la innovación social.

\section{Economía social y economía colaborativa}

Como se ha apuntado anteriormente, la llamada economía colaborativa supone un cambio de paradigma respecto al modelo que podría denominarse economía tradicional; si bien puede apreciarse un cierto paralelismo entre este modelo emergente y el movimiento cooperativo, asimilando los conceptos de “colaboración” y "participación”. Según García-Gutiérrez Fernández (1988: 105-106): “Es importante tener en cuenta que el trabajador de una empresa de socios-trabajadores no es un asalariado que deba atenerse a la estructura jerárquica de autoridad por razones de eficiencia; sino que dentro de su marco de autonomía, por pequeño que sea, tiene que procurar ser lo más eficiente que sea posible y actuar como un auténtico empresario: innovando, emprendiendo, ideando, mejorando, acometiendo proyectos supuestamente eficientes. De tal manera que es aquí, en el puesto de trabajo, donde se produce la participación".

Como requerimientos para esa participación política o co-gobernanza, que se traduce en la toma de decisiones y en fijar objetivos, aparece en primer lugar el tener acceso a una buena y adecuada información, así como a canales de comunicación eficientes que garanticen la transparencia informativa. De esta forma, la información y la comunicación se convierten no en una simple herramienta de gestión para transmitir de forma unidireccional las decisiones tomadas desde un nivel superior jerárquico de la organización, sino en los presupuestos para una auténtica participación y colaboración. Esta debería ser la seña identitaria de las organizaciones cooperativas, porque, como recuerda García-Gutiérrez Fernández (1988: 38): "En la empresa cooperativa tiene que haber participación en la toma de decisiones; ya que, en definitiva, es una empresa de empresarios, de participadores en los flujos reales, y en los flujos financieros, y en los flujos de informacióndecisión". 
Iturrioz del Campo (2001) apunta que la participación de los socios en los flujos empresariales es uno de los criterios que permite clasificar los diferentes tipos de empresas. Por tanto, podrían definirse como empresas de participación a aquellas en las que los socios participan activamente en el proceso de producción o distribución o actúan como consumidores finales.

No obstante, debe añadirse una segunda condición al concepto de empresa de participación para asegurar que la participación de los socios suponga una diferenciación en su funcionamiento. Esta segunda condición sería que, al menos, la mayoría del poder de decisión se encuentre en manos de los socios que participan en los tres flujos empresariales. De ahí que "se pueda diferenciar entre dos conceptos de empresa de participación; el concepto amplio (en el que se incluyen las empresas cuyos socios participan en los tres flujos empresariales) y el concepto restringido (en el que además los citados socios deben tener la mayoría del poder de decisión)" (Iturrioz del Campo, 2001:152). Entre las empresas que cumplen estas condiciones del concepto restringido se encuentran muchas vinculadas con la economía social, entre ellas las sociedades cooperativas, las mutuas o las sociedades laborales anónimas o de responsabilidad limitada.

Estas entidades de economía social tienen su reconocimiento legal a partir de una recomendación del Parlamento europeo en 2009, que pedía a la Comisión y a los Estados miembros que regularan la economía social desde un enfoque diferente al de la empresa; considerando que la finalidad principal de estas entidades no es la rentabilidad financiera sino los beneficios para toda la sociedad.

Desde esta perspectiva se aprueba en España la Ley de Economía Social 5/2011, con el objetivo de configurar un marco jurídico que diera visibilidad y a la vez promoviera la economía social, dotándole de una mayor seguridad jurídica. Esta ley en su artículo 2 define la economía social como "conjunto de las actividades económicas y empresariales, que en el ámbito privado llevan a cabo aquellas entidades que, de conformidad con los principios recogidos en el artículo 4, persiguen bien el interés colectivo de sus integrantes, bien el interés general económico o social, o ambos".

Como señala Fajardo García (2018), esta definición de economía social no termina de aclarar qué entidades podrían entrar en este modelo, más allá de las que taxativamente enumera. Entre otras cuestiones, establece como elemento definitorio un conjunto de actividades económicas y empresariales, cuando no son las actividades por sí mismas las que identifican a la economía social, sino la finalidad perseguida con las mismas y la forma en que se desarrollan, para lo cual el legislador remite al artículo 4.

Adicionalmente, convendría precisar que esas actividades, para considerarse dentro del ámbito de la economía social, deben ser desarrolladas con criterios empresariales, por entidades privadas con personalidad jurídica, excluyendo de esta forma las actividades similares que pudieran desarrollar personas físicas o entidades públicas.

Por último, esta definición legal refiere que en su actuación esas entidades persiguen ciertos fines y tienen como fundamento ciertos principios, ya comentados en la exposición de motivos de la ley; pero dichos fines y principios se expresan con una redacción ambigua, lo que dificulta su comprobación, generando una cierta inseguridad jurídica, que es precisamente lo que se trataba de evitar con la aprobación de esta ley.

Probablemente la intención del legislador de enmarcar o definir un modelo en constante evolución le haya impulsado a intentar concretar en un primer momento, para luego dejar la puerta entreabierta a la inclusión de otras entidades que, sin ser las prototípicas de este modelo, como por ejemplo las cooperativas, puedan tener cierta afinidad con sus principios y fines. El resultado es que no se ha conseguido ni lo uno ni lo otro, con la consecuente inseguridad jurídica que se deriva de esta cierta indefinición.

Una cuestión más de fondo es la decisión del legislador de no adoptar en su totalidad los principios que consensuada e internacionalmente identifican a las entidades de la economía social; a la vez que incorpora otros que no son exclusivos de estas entidades. Añade de esta forma mayor confusión, y dificulta la interpretación y aplicación de los principios que deben caracterizar, o al menos orientar, la actividad de esas entidades.

Como acertadamente señala Fajardo García (2018:111) "Los principios de la economía social internacionalmente reconocidos son por lo general principios estructurales, están integrados en la estructura de la entidad por imperativo legal: la primacía de la persona y del objeto social sobre el capital; la adhesión voluntaria y abierta; el control democrático por sus miembros; la conjunción de los intereses generales con los intereses de los miembros usuarios; la autonomía de gestión e independencia de los poderes públicos, o la forma de distribuir los resultados, se corresponden con normas que regulan estas formas jurídicas. Estos principios se corresponden en buena medida con los llamados principios cooperativos, que a su vez se inspiran en normas propias de las asociaciones y mutualidades. Estos principios están presentes estructuralmente en las entidades clásicas de la economía social y muchos de ellos también en aquellas otras entidades con las que comparten fines, formas organizativas y pautas de comportamiento. (...) Por el contrario, principios como los que incorporan los objetivos de la RSE son principalmente conductuales".

Se refiere aquí a la responsabilidad social empresarial (RSE), por cuanto la reciente Estrategia Española de la Economía Social 2017-2020 define a las entidades de economía social como empresas socialmente responsables y establece diversas medidas para impulsar la RSE en el marco de estas entidades; a la vez que reitera el compromiso de extender la Estrategia de la Responsabilidad Social a todas las empresas. Un 
objetivo también compartido por la Ley 2/2001 sobre Economía Sostenible que tienen por objeto "incentivar" a las empresas a incorporar o desarrollar políticas de responsabilidad social, definida en el Informe de la Subcomisión Parlamentaria para promover la RSE como "la integración voluntaria por parte de la empresa, en su gobierno y gestión, en su estrategia, políticas y procedimientos, de las preocupaciones sociales, laborales, ambientales y de respeto a los derechos humanos...".

Coincidimos con Fajardo García (2018:109) al señalar que "hay principios que caracterizan a las entidades de la economía social, que han sido reconocidos internacionalmente, y que también están presentes en el artículo 4, sin excluir el principio de adhesión voluntaria y abierta; y hay otros principios que son orientadores de la actividad de las entidades de la economía social como son los objetivos de la RSE. Estos, si bien no deberían ser exigibles para acceder al catálogo de entidades de la economía social, si pudieran serlo a la hora de beneficiarse de políticas de promoción de la economía social, si es que quiere promoverse su cumplimiento".

En definitiva, al plasmarse legalmente de esta forma la definición de economía social puede haberse en realidad retrocedido respecto a las diversas definiciones que con anterioridad la doctrina había propuesto, siendo la más reconocida la que recoge el Informe elaborado por los profesores Chaves y Monzón (2008, citados por Fajardo García, 2018:101) para el Comité Económico y Social Europeo titulado "La Economía Social en la Unión Europea". Este Informe formula una definición operativa de la Economía Social que permita cuantificar y hacer visible de forma homogénea y armonizada internacionalmente, los principales agregados de las entidades que la integran. Según esta definición, la economía social es el "Conjunto de empresas privadas organizadas formalmente, con autonomía de decisión y libertad de adhesión, creadas para satisfacer las necesidades de sus socios a través del mercado, produciendo bienes y servicios, asegurando o financiando y en las que la eventual distribución entre los socios de beneficios o excedentes así como la toma de decisiones, no están ligados directamente con el capital o cotizaciones aportados por cada socio, correspondiendo un voto a cada uno de ellos. La Economía Social también agrupa a aquellas entidades privadas organizadas formalmente con autonomía de decisión y libertad de adhesión que producen servicios de no mercado a favor de las familias, cuyos excedentes, si los hubiera, no pueden ser apropiados por los agentes económicos que las crean, controlan o financian".

Sin embargo, no consideramos que sea esta una cuestión meramente doctrinal o teórica. La desviación o directamente la ausencia de estos principios y fines en las actividades de las entidades de economía social no solo sería un dato relevante para clasificarlas de una u otra forma; lo relevante aquí es que al alejarse de estos fines y principios pierden sus ventajas comparativas como modelo alternativo a la economía tradicional y de esta forma se ven arrastradas por las mismas circunstancias, y en igual medida, que las empresas de corte capitalista.

En un reciente trabajo (García, M., 2017), se compara la evolución durante la crisis de la economía general con la economía social utilizando algunos indicadores. La hipótesis inicial era esperar un mejor comportamiento de la economía social ante la crisis, debido a que esta última debía ser más intensiva en trabajo que la general, aunque de ello se derive una menor productividad. Al mismo tiempo, se supone que en estas entidades prima la persona sobre el beneficio empresarial, por lo que cabría esperar mejores datos de empleo y salarios que en las empresas mercantiles y una mayor flexibilidad en aras de al menos conservar los puestos de trabajo. Sin embargo, respecto a los datos de empleo, el número de empresas y el resto de las variables estudiadas, el comportamiento de la economía social es análogo que el de la general. Como conclusión del estudio, se establece que en una situación de crisis parece que la economía social no tiene un mejor funcionamiento que la economía general. Esto es contrario a lo que teóricamente se presupone es la principal ventaja comparativa de las entidades de economía social, que es precisamente su capacidad de resiliencia para adaptarse a entornos económicos adversos, manteniéndose como un cierto nicho de empleo, al menos localmente.

Otra conclusión de los datos estadísticos aportados por el anterior estudio es que las entidades de economía social, a pesar de lo formulado en sus fines y objetivos, en la práctica muchas veces siguen modelos de gestión similares a las empresas capitalistas. Por ello no es de extrañar que la propia crisis global hiciera emerger nuevos modelos económicos, aún más descentralizados y horizontales, en el marco de lo que se ha denominado economía colaborativa.

El nacimiento de este modelo emergente puede situarse, precisamente, en medio de la crisis económica global, a partir del 2010, cuando se comienza a hablar de consumo colaborativo, concepto que posteriormente evoluciona hacia el de economía colaborativa. Para Gansky, $(2010)^{2}$; lo esencial de esta

\footnotetext{
2 "What characterizes a Mesh business?

1. The core offering is something that can be shared, within a community, market, or value chain, including products, services, and raw materials.

2. Advanced Web and mobile data networks are used to track goods and aggregate usage, customer, and product information.

3. The focus is on shareable physical goods, including the materials used, which makes local delivery of services and products-and their recoveryvaluable and relevant.

4. Offers, news, and recommendations are transmitted largely through word of mouth, augmented by social network services.
} 
nueva forma de hacer negocios es la interconexión de todos los miembros de la red, que se puede hacer extensiva a nuevos agentes; pero ya desde entonces señala como elemento esencial de este nuevo paradigma el uso compartido de los bienes, tanto de las materias primas como de los productos finales. Para designar a este modelo de negocios habla de "Mesh business", donde el término "mesh" (malla) se refiere a cómo la tecnología digital se usa para proporcionar bienes y servicios de nuevas y variadas formas. Describe así una forma totalmente nueva de servicios basados en la información y que se pone de evidencia en fenómenos como Facebook y Twitter.

Botsman y Rogers, (2010), definen lo que denominan "consumo colaborativo" también como un nuevo modelo de economía, analizando para ello otros casos de éxito como Zipcar, eBay, Airbnb y Wikipedia. Los propios autores establecen algunos principios generales para la conceptualización de este modelo, entre ellos la compatibilidad entre la eficiencia empresarial y la protección del medio ambiente. Sostienen que dichos objetivos estarán alineados cuando se cambie el paradigma dominante en el mundo económico, maximizar la producción y venta de unidades; por otro en que se busque incrementar el número de veces en que se pueda usar un producto. Como consecuencia de lo anterior se alcanzaría una redistribución de la riqueza, una etapa a la que denominan la quinta "R" porque vendría como lógica continuación de reducir (la producción), reciclar, reutilizar y reparar ("reduce, recycle, reuse, repair and redistribute"). Finalmente, postulan que el acceso es mejor que la propiedad, una especie de axioma que tendrá desarrollo en la economía colaborativa.

La fecha en que surgen estos conceptos es muy significativa, en plena crisis económica y financiera. En ocasiones se pone el foco en que la motivación y causa de este nuevo modelo es el consumo responsable y la mayor sensibilidad medioambiental, lo que seguramente es cierto en muchos casos, pero no debe obviarse otros factores como la crisis financiera y el desarrollo de internet, que por un lado fuerzan la búsqueda de nuevas formas de organización, muchas veces orientadas al autoempleo, y por otro permiten modelos alternativos más eficientes, basados en plataformas colaborativas.

Los desafíos que esto plantea son muchos y se extienden a muy diversos campos. Desde el punto de vista regulatorio, incrementar la confianza del consumidor pasa por regular los derechos y deberes de las partes que interaccionan en estas plataformas, con especial atención a los derechos de los consumidores, pero la cuestión se complica cuando no hay consenso en qué requisitos debería tener el usuario para ser considerado consumidor y por tanto disfrutar de esa especial protección. La creación de una base de datos de clientes y proveedores también supone un reto para la protección de los datos personales, porque con la actual regulación en ocasiones no se puede establecer con claridad quién es el responsable del tratamiento de los datos (el responsable de la plataforma o quien presta el servicio, por ejemplo) en qué condiciones se ceden, quienes tendrían acceso a ellos, etc. En el caso de algunas plataformas habría que definir cuándo el particular adquiere la condición de profesional o empresario, con los deberes que ellos supone, incluidos los de tipo tributario; y buscar un necesario equilibrio entre la utilización de estas plataformas para reducir el coste económico de las transacciones, pero a la vez prevenir la evasión fiscal, porque su deslocalización y opacidad pueden ser una tentación para empresas y particulares que adopten este modelo con el único propósito de defraudar o evadir impuestos.

Es, por tanto, la economía colaborativa en la actualidad un fenómeno complejo que engloba bajo un mismo término varias realidades. Una vez rebasado por insuficiente el concepto original de consumo colaborativo, se ha intentado perfilar sus elementos, pero la mayor claridad ha supuesto en muchos casos una visión reduccionista, como cuando se utiliza como sinónimo el término "economía entre pares" (peer - P2Peconomy). En este caso, solo entrarían en el concepto de economía colaborativa los modelos de negocio peer-to-peer, en el que las plataformas se usan para alquilar, vender, prestar o compartir bienes al margen de tiendas, bancos o agencias; pero de esta forma se excluirían otras fórmulas entre empresarios y consumidores, empresarios y empresarios o, incluso, entre consumidores y empresarios.

Díaz, Marcuello y Monreal (2016) presentan varias definiciones, desde las iniciales de Botsman y Rogers (2010), que resaltan su construcción como modelos alternativos, como prácticas y modelos de negocio basados en redes horizontales y la participación de una comunidad, en lugar de instituciones centralizadas. También citan una definición de la Revista Opciones (2013): "Un movimiento que engloba nuevas prácticas económicas que tienen en común algún grado de participación u organización colectiva en la provisión de bienes y servicios". Una tercera definición habla de un modelo en el que se borran los límites entre productores y consumidores, que interactúan en internet, en plataformas peer-to-peer (P2P) y en otras experiencias offline (Ouishare, 2016).

Why call this new wave of businesses "The Mesh"? A Mesh describes a type of network that allows any node to link in any direction with any other nodes in the system. Every part is connected to every other part, and they move in tandem. To me, "The Mesh" is an apt and rich metaphor to describe a whole new phase of information-based services. Mesh businesses are knotted to each other, and to the world, in myriad ways. Some connections are formed directly, such as an agreement among companies to identify a market and make coordinated offers. These companies share information to facilitate access to new customers, customer preferences, and goods. Other connections are formed indirectly through third parties, such as aggregated consumer data or via customers' social networks." 
Mientras las dos primeras ponen el énfasis en la comunidad, la tercera definición apunta a las implicaciones y consecuencias que la irrupción de esta nueva red horizontal tiene sobre el modelo económico clásico. Es lógico que, si en el marco de esa comunidad las interacciones son esencialmente de intercambio (y no de venta), ambas partes serían mutua y recíprocamente productores y consumidores, en contraposición al modelo mercantil tradicional, en el que una empresa, actuando como productor, suministra bienes o servicios a un consumidor.

Es, sin embargo, la definición de Sharing España, la asociación que agrupa a las grandes empresas de este sector en nuestro país, la que parece aportar mayor claridad, a la vez que amplitud y matices sobre el fenómeno aún en crecimiento de la economía colaborativa. Para esta asociación se trata de "nuevos modelos empresariales y de consumo en los que gracias a las nuevas tecnologías se accede a bienes y servicios más eficientes y participativos basados en la comunidad y la confianza, así como en la oferta basada en el acceso al bien improductivo en contraposición a su adquisición; combinación que unas veces puede basarse en una relación entre iguales (P2P) o bien en la puesta a disposición por parte de una empresa al acceso por los usuarios de bienes bajo demanda cuando les resulte más conveniente (B2P)" (SHES, 2016, en Díaz, Marcuello y Monreal, 2016: 28).

Esta cuarta conceptualización pone el foco en un elemento esencial: el acceso a bienes y servicios, nuevamente en oposición al tradicional cambio de titularidad por la transmisión de la propiedad. No se trata entonces de encuadrar o excluir de la economía colaborativa los diversos modelos en función de aspectos formales como la consideración de empresa o particular que tengan sus integrantes, sino de atender a lo que representa el objetivo esencial por el que interactúan a través de las plataformas, esto es, a si su finalidad es la comercialización o por el contrario la satisfacción de necesidades colectivas de la comunidad a través del acceso y uso compartido de los bienes y servicios.

Según Alfonso (2016:236), existe un cierto consenso en la doctrina respecto a los rasgos distintivos de la economía colaborativa, entre los cuales cita: "a) el necesario uso de internet; b) la interconexión en red de personas y/o activos; c) el acceso al uso de activos tangibles e intangibles desaprovechados; d) el logro de interacciones significativas y la confianza; y e) el carácter abierto, inclusivo y global".

A partir de estos rasgos generales puede establecerse una clasificación atendiendo a cuál sea el objetivo principal perseguido por las empresas de economía colaborativa, en función de, al menos, cuatro actividades básicas.

La primera de ellas es el consumo colaborativo, que se fundamenta en el acceso a los bienes y/o servicios a través de instrumentos jurídico-económicos tales como bartering, renting, lending, trading, leasing, exchanging, reselling y swapping. Incluye tanto los mercados de redistribución, los sistemas de producto y servicio, en los que se paga por el acceso a los bienes y no por su adquisición; así como los sistemas de vida colaborativa, donde se comparten e intercambian activos intangibles, como tiempo y habilidades.

La segunda actividad sería la referida a la producción colaborativa, en la que grupos, redes o particulares colaboran en el diseño, producción o distribución de bienes. Incluye el diseño colaborativo, cuyas características principales ya habían sido apuntadas por Gansky $(2010)^{3}$.

La tercera comprende el aprendizaje colaborativo, que incluye el acceso libre y gratuito a cursos, lecturas y contenidos educacionales, open courses y courseware, (FutureLearn, Moocs, Coursera), así como la enseñanza de destrezas, skillsharing (Skilio), o la aportación pública de conocimientos o de resolución de problemas (Wikipedia); mientras que la cuarta actividad tiene que ver con las finanzas colaborativas, o servicios de financiación, préstamo e inversión que se prestan al margen de las entidades financieras tradicionales.

En los últimos años también se habla de una quinta actividad, la gobernanza colaborativa, que hace referencia a nuevos mecanismos de gobernanza horizontal y participativa que tienen lugar tanto en las ciudades como en las empresas (Alfonso, 2016). En el primer caso, el compromiso cívico se expresa en términos colaborativos entre los funcionarios públicos, el sector privado, los ciudadanos y las organizaciones civiles para la co-creación de métodos, técnicas y habilidades, haciendo uso de las tecnologías de información y comunicación. La finalidad de esta innovación pública es mejorar la eficiencia y la calidad de los procesos, la dinámica organizacional y los flujos de la información en la comunicación en las organizaciones públicas y de éstas con la ciudadanía, así como la producción y provisión de los servicios con el objetivo de lograr valor, expresado en índices de satisfacción. Para todo ello es esencial contar con una

\footnotetext{
${ }^{3}$ In a Mesh business, products are shared. The flow of information about the products, including feedback from customers, is constant. As a result, favored products are built to last and keep functioning, adapt to different users, and be capable of repair and upgrading. The logic of the throwaway culture is completely reversed. Mesh design is:

Durable. Products that many people use must be safe, well built, and longer lasting.

Flexible. Products accommodate different users with design that is modular, but easily personalized.

Reparable. Standardized parts and transparent design allow products to be fixed and reused, rather than trashed, encouraging a culture of repair and reuse.

Sustainable. Design that reduces natural resource destruction and waste, which is ever more expensive, improves efficiency and reduces overall costs. (Gansky, 2010: 35).
} 
plataforma colaborativa digital, que facilite el compartir información y la interacción, a la vez que garantice la transparencia propia de los modelos de e-gobierno o administración electrónica (Mariñez, 2016).

En el Informe sobre una Agenda Europea para la economía colaborativa, de 11 de mayo de 2017, de la Comisión de Mercado Interior y Protección del Consumidor, se propone una definición muy general, incluyendo una gama de actividades derivadas de distintos modelos de negocio colaborativos, en particular aquellos sin ánimo de lucro; dichos modelos van desde las plataformas colaborativas que permiten el intercambio de bienes y servicios en todo el mundo a las pequeñas pymes colaborativas que prestan servicios a sus comunidades.

Dicho informe constata que en los últimos años ha habido un rápido crecimiento de la economía colaborativa en cuanto a número de usuarios, transacciones e ingresos, lo que ha acabado reconfigurando la manera en que se ofrecen productos y servicios en numerosos ámbitos. A principios de 2014 , los ámbitos de actividad que habían resultado más afectados solo eran seis (bienes y comercio al por menor; servicios; espacio y alojamiento; alimentación; transporte, y dinero), mientras que en 2016 se había prácticamente triplicado el número de ámbitos marcados por estos cambios, ya sea por el contagio a otros sectores (por ejemplo, salud, aprendizaje, logística, municipios, espacio, servicios públicos como electricidad, etc.) o la creación de nuevos tipos de actividades (es decir, el movimiento maker aplicado al sector de bienes).

Según otro reciente informe (Vaughan y Daverio, PwC UK, 2016), se estima que se han creado más de 275 organizaciones de economía colaborativa en los nueve mayores estados miembros de la Unión Europea. La lista la encabezaba un ya exmiembro, el Reino Unido, que junto a Francia aportaban al cómputo global cerca de 50 organizaciones. En el segundo grupo entraban Alemania, España y Holanda, con una contribución de 25 organizaciones.

Para el caso de España, las plataformas de alquiler vacacional $P 2 P$ ocupaban el primer lugar en popularidad, estimándose que eran las responsables de un $25 \%$ del total de plazas turísticas, llegando hasta casi el 50\% en el caso de las ciudades con mayor interés turístico, superando en número a la oferta hotelera en esas mismas zonas. Las tres mayores plataformas de alquiler vacacional abarcaban un $65 \%$ de este sector, en primer lugar Airbnb con un 27\%, seguida por HomeAway con un 24\% y Niumba con el $14 \%$.

Por otra parte, las plataformas de finanza colaborativa mostraban un importante incremento como alternativa a la banca tradicional, situando a España en el sexto lugar a nivel europeo, con un volumen de transacciones cercano a 62 millones de euros.

En el conjunto de Europa, y pese a la extensión de la economía colaborativa en todos los sectores, hasta el año 2015 era el sector transportes con 1.650 millones de euros el que había registrado más ingresos, si bien la tendencia apunta a que el sector de alojamiento con un total de 1.150 millones de euro termine superándole (Ostelea, 2018, gráfico 1).

Gráfico. 1. Ingresos por ámbitos de economía colaborativa en Europa, 2015. Fuente: Statista 2017, en Ostelea (2018).

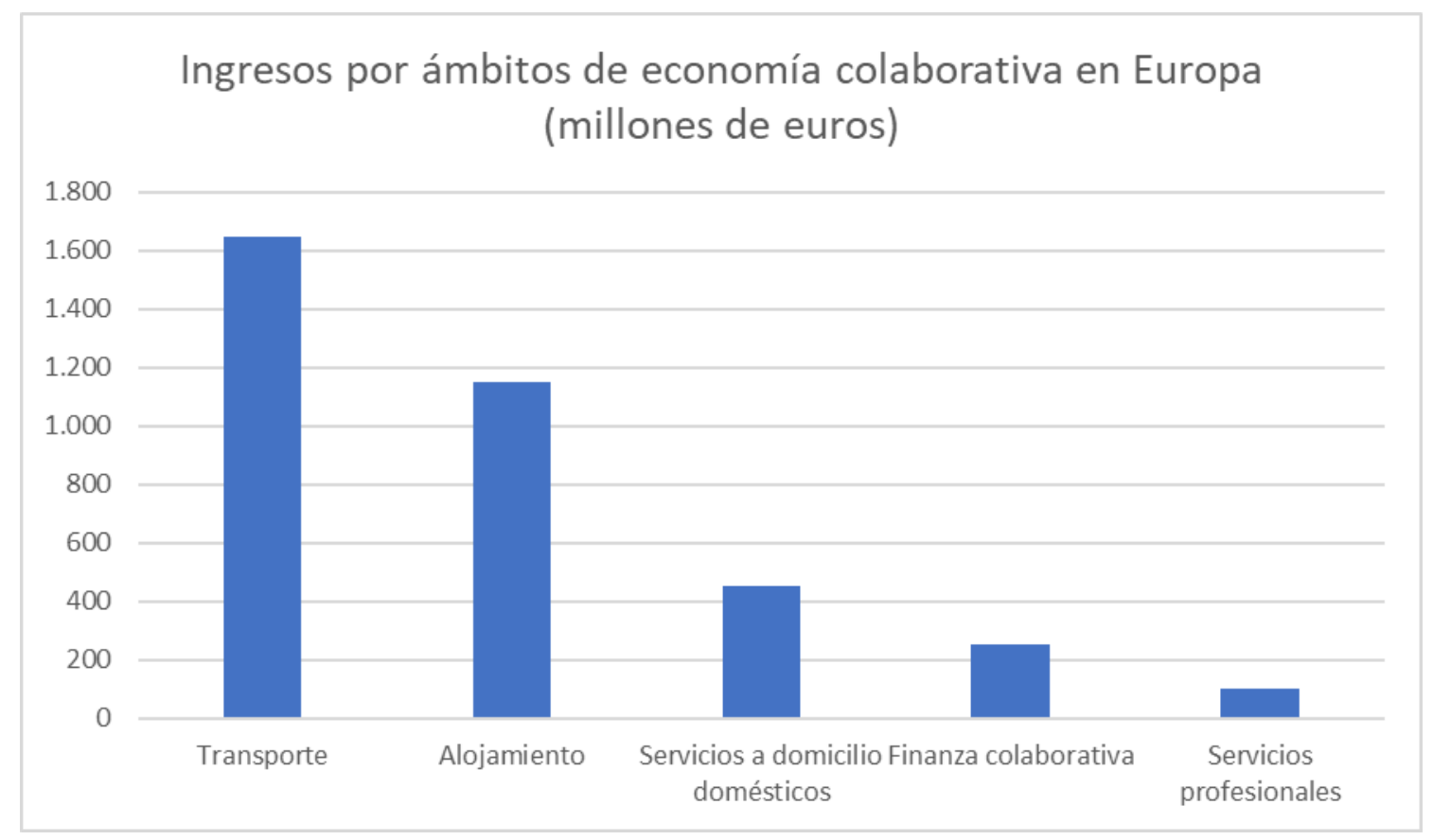

A la dificultad de poner límites conceptuales a un fenómeno aún en rápido crecimiento, la Comisión Europea añade otra y es los diferentes términos con un sentido similar y a menudo intercambiables, a los que se recurre para describir este fenómeno: economía participativa, economía inter pares, economía a la 
demanda, economía de bolos, consumo colaborativo o capitalismo participativo, entre otros. Estas definiciones reflejan tanto la dinámica del fenómeno en sí como la magnitud e intensidad del debate político y académico que tiene lugar al respecto.

En el referido Informe sobre una Agenda Europea para la economía colaborativa se cita una definición del diccionario Oxford, para el cual la economía colaborativa se trata de un sistema económico en el que los bienes o servicios son compartidos por particulares, ya sea gratuitamente o previo desembolso, generalmente a través de internet. Por su parte, la Comisión Europea la define como modelos de negocio en los que se facilitan actividades mediante plataformas colaborativas que crean un mercado abierto para el uso temporal de mercancías o servicios ofrecidos a menudo por particulares. Igualmente precisa que, por lo general, las transacciones de la economía colaborativa no implican un cambio de propiedad y pueden realizarse con o sin ánimo de lucro.

Dentro de la diversidad de modelos de negocio, se suelen distinguir tres grandes categorías de agentes:

i. los prestadores de servicios, particulares que ofrecen servicios (activos, competencias o tiempo, ya sea gratuitamente o previo pago) de manera ocasional ("pares") o a título profesional ("profesionales");

ii. los usuarios de dichos servicios, $\mathrm{y}$

iii. las plataformas colaborativas que conectan a la oferta con la demanda en tiempo real y facilitan las transacciones.

Una clasificación frecuente es la que tiene en cuenta tanto los prestadores de servicios como a los usuarios de estos. Se distingue así el modelo B2C o Business-to-Consumer, en el que la empresa es titular del servicio o la prestación; el $\mathrm{B} 2 \mathrm{~B}$ o Business-to-Business, donde la interacción se realiza entre empresas que poseen o manejan el servicio o la prestación; el P2P o Peer-to-Peer, en el que son los particulares los que a través de la plataforma interactúan y cambian o realizan las prestaciones o servicios; el C2B o Consumer-toBusiness en el que la empresa se beneficia y paga por los conocimientos o activos de los consumidores; y el G2G o Government-to- Government en el que son las administraciones públicas los que interactúan a través de la plataforma.

Otra clasificación (Alfonso, 2016), dibuja cuatro ejes y ocho características de la posibilidad de "compartir":

1) formal/informal sharing, según la actividad se realice en el marco de un sistema legal formal o al margen del aparato regulador, pero sin llegar a la ilicitud; es decir, adoptando algunas de las fórmulas ya previstas (p. e. renting) o bien otras jurídicamente atípicas. 2) gratuitous/nongratuitous sharing, dependiendo de que el servicio se preste con la finalidad de obtener una ganancia o una contraprestación; 3) monetary/nonmonetary sharing, en función de que la actividad implique o no intercambio de dinero; y 4) commercial/personal sharing, esta última de difícil concreción porque la distinción entre "pares" o profesionales, o lo que es lo mismo, cuándo puede considerarse que quien presta los servicios tiene la condición de empresario, es una cuestión que presenta notables lagunas en nuestro marco jurídico.

Esto es así porque los ciudadanos se convierten en agentes económicos, capaces de generar e intercambiar valor con otros ciudadanos de manera directa. En muchos casos realizarán estos intercambios por dinero (alquiler, compraventa), aunque el abanico de posibilidades incluye también el intercambio (bien sea directo o triangulado a través de alguna "moneda social" o sistema similar) y el regalo o préstamo gratuito. (Helms y Palacios, 2016; en Cañigueral, 2016).

Por último, varias clasificaciones atienden al papel que juega la plataforma colaborativa, diferenciando, por ejemplo, entre plataformas comerciales o for-profit (FP) y plataformas not-for-profit (NFP). Mayor interés reviste la clasificación que atiende al comportamiento de la plataforma, es decir, si lo que permite o posibilita ésta es la creación de un marco de oferta y demanda de productos y servicios entre proveedores y usuarios, convirtiéndose así la propia plataforma en un marco de intercambio, o si aquello a lo que da lugar la plataforma es a una organización (empresa o entidad gubernamental).

Es posible que en algunos modelos la plataforma se limite a hacer de intermediario digital, una función propia de un prestador de servicios de la sociedad de la información, poniendo en contacto a los prestadores/proveedores con los usuarios y facilitando la colaboración entre ellos. Esta actividad, por sí misma, no es lo que se considera economía colaborativa, sino que es una actividad mercantil de intermediación que favorece la puesta en contacto entre usuarios para que sean ellos los que puedan llevar a cabo las actividades de la economía colaborativa propiamente dichas.

Será, por tanto, la actividad que llevan a cabo los usuarios de esa plataforma, la actividad subyacente, la que sí que tiene que ver con la economía colaborativa o bajo demanda. Son estos usuarios los que intercambian su tiempo, conocimiento o bienes infrautilizados a cambio de una contribución a los gastos, principalmente en los ejemplos de economía colaborativa, o se prestan el servicio de que se trate y se pagan el precio convenido, en el caso de la economía bajo demanda.

Las plataformas muchas veces son mercados multilaterales, donde los usuarios interactúan y llevan a cabo transacciones entre ellos, por lo que pueden adoptar el papel de proveedor, de consumidor, o de ambas 
figuras ("prosumidor"). Sin embargo, no hay que obviar que estas plataformas también pueden servir como vías adicionales o alternativas de negocio para prestadores tradicionales en el marco de la economía bajo demanda. (SHES, 2017)

\section{Las plataformas digitales}

Las plataformas son esenciales en la configuración de los mercados de la economía colaborativa y desarrollan diversas funciones, entre ellas conectar a quienes ofrecen un activo con quienes desean acceder a él, facilitar que la transacción económica se realice por vía electrónica y proveer los mecanismos de verificación que conformen la reputación online e incremente la confianza de los usuarios. Si la plataforma funciona dentro de estos límites tendrá la condición de intermediario digital, si se excede de ello podría considerarse que es la propia plataforma quien presta el servicio y, por tanto, entra en competencia con los agentes tradicionales (Alfonso, 2016). La jurisprudencia europea pone especial atención en determinar si la plataforma tiene capacidad de organización, lo que supone un cierto control sobre los precios y la forma en que se presta el servicio, estableciendo ciertos parámetros o elementos esenciales de la prestación.

Otra función esencial de la plataforma en la economía colaborativa es su papel en la construcción de la comunidad que interactúa a través de ella. Se trata de un vínculo que rebasa la mera relación económica o mercantil, se trata de relaciones de confianza que se fundamentan muchas veces en objetivos compartidos. Sin embargo, el que no exista una plataforma digital no excluye a algunos modelos de la economía colaborativa, porque también esta se puede configurar de forma offline. Son claros ejemplos de ello los bancos del tiempo, los grupos de consumo, los huertos urbanos, los espacios makers, fablabs o los coworkings. En el sentido opuesto, el que exista una plataforma colaborativa que ponga en contacto a usuarios y proveedores no significa necesariamente que estemos en un marco de economía colaborativa.

Para Sharing España (2016), se pueden distinguir al menos tres modelos económicos que comparten el uso de plataformas digitales. Es así que define a la economía colaborativa como "aquellos modelos de producción, consumo o financiación que se basan en la intermediación entre la oferta y la demanda generada en relaciones entre iguales (P2P o B2B) o de particular a profesional (P2B), como en el caso del crowdfunding a través de plataformas digitales que no prestan el servicio subyacente, generando un aprovechamiento eficiente y sostenible de los bienes y recursos ya existentes e infrautilizados, permitiendo utilizar, compartir, intercambiar o invertir los recursos o bienes, pudiendo existir o no una contraprestación entre los usuarios (SHES, 2016: 9).

Señala igualmente que el término "recurso infrautilizado" debe interpretarse de manera amplia, entendido como recurso no solamente un bien, como un vehículo o un inmueble, sino también el tiempo, el conocimiento o incluso el dinero. En cuanto a la contraprestación, en el caso de que exista, puede darse como actividades sin ánimo de lucro en las que se comparten únicamente los gastos ocasionados por un bien puesto en valor y también actividades con ánimo de lucro, como la compraventa de segunda mano entre particulares.

En un sentido opuesto, la economía bajo demanda serían "aquellos modelos de consumo y provisión de servicios que se basan en la intermediación entre la oferta y la demanda generada habitualmente de profesional a consumidor (B2C) a través de plataformas digitales que no prestan el servicio subyacente y cuya prestación se origina en base a las necesidades del usuario que demanda y se adapta a sus preferencias, prestándose normalmente a cambio de una contraprestación y habitualmente con ánimo de lucro.

La diferencia fundamental de este tipo de modelos bajo demanda y los modelos colaborativos es que entre los usuarios existe una relación comercial, es decir, son plataformas en las que tiene lugar la prestación de un servicio ya sea por parte de profesionales o por parte de particulares, dependiendo del modelo.

Finalmente, formarían parte de la economía de acceso aquellos modelos de consumo en los cuales una empresa, con fines comerciales, pone a disposición de un conjunto de usuarios unos bienes para su uso temporal, adaptándose al tiempo de uso efectivo que requieren dichos usuarios y flexibilizando la localización espacial de los mismos.

Si bien la economía colaborativa ya fomenta el acceso frente a la propiedad de los bienes, la economía de acceso hace referencia únicamente a los modelos en los que la plataforma digital sí presta el servicio subyacente y los usuarios normalmente no tienen contacto directo entre sí para efectuar las transacciones" (SHES, 2016:9).

Parece contradictorio que en el citado documento Sharing España afirme que "los casos más paradigmáticos de la economía de acceso son los modelos de carsharing o los coworkings" (SHES, 2016:9) cuando previamente había citado la Sentencia N. ${ }^{\circ}$ 30/2017 de 2 de febrero de 2017, del Juzgado de lo Mercantil n. ${ }^{\circ} 2$ de Madrid, en el caso Confebus contra la plataforma www.blablacar.es, en la que además de absolver a la acusada, confirmaba la aplicación de la Directiva de Comercio Electrónico y la LSSI a las plataformas de la economía colaborativa, confirmando igualmente que queda probado que Blablacar realiza una actividad ajena a la regulada por la LOTT, pues poner en contacto a particulares con más o menos 
requisitos, con un control de pagos, con una crítica de las personas intervinientes sobre retrasos o sobre la calidad de otros servicios, no es una actividad sujeta a la ley de ordenación del transporte terrestre.

Seguidamente, el juez destaca que "Blablacar ha generado una plataforma no para organizar el transporte, sino para poner en contacto a particulares que quieren realizar un viaje juntos, y compartir determinados gastos del trayecto". La contradicción reside, no en que Blablacar sea un modelo de economía de acceso, sino en el concepto que refiere sobre aquella, en el que se subraya expresamente que, en este caso, la plataforma digital sí presta el servicio subyacente.

Un aspecto relevante por considerar es la motivación de los usuarios o consumidores para utilizar estas plataformas colaborativas. En un reciente informe (ING internacional, 2015) se preguntaba a una muestra de 14.829 personas sobre la posible influencia de cuatro factores para la participación en la economía colaborativa (gráfico 2).

Gráfico. 2. Factores que influyen en la decisión de participar en la economía colaborativa. Fuente: elaboración propia a partir del informe "The Sharing Economy 2015" (ING International, Julio 2015).

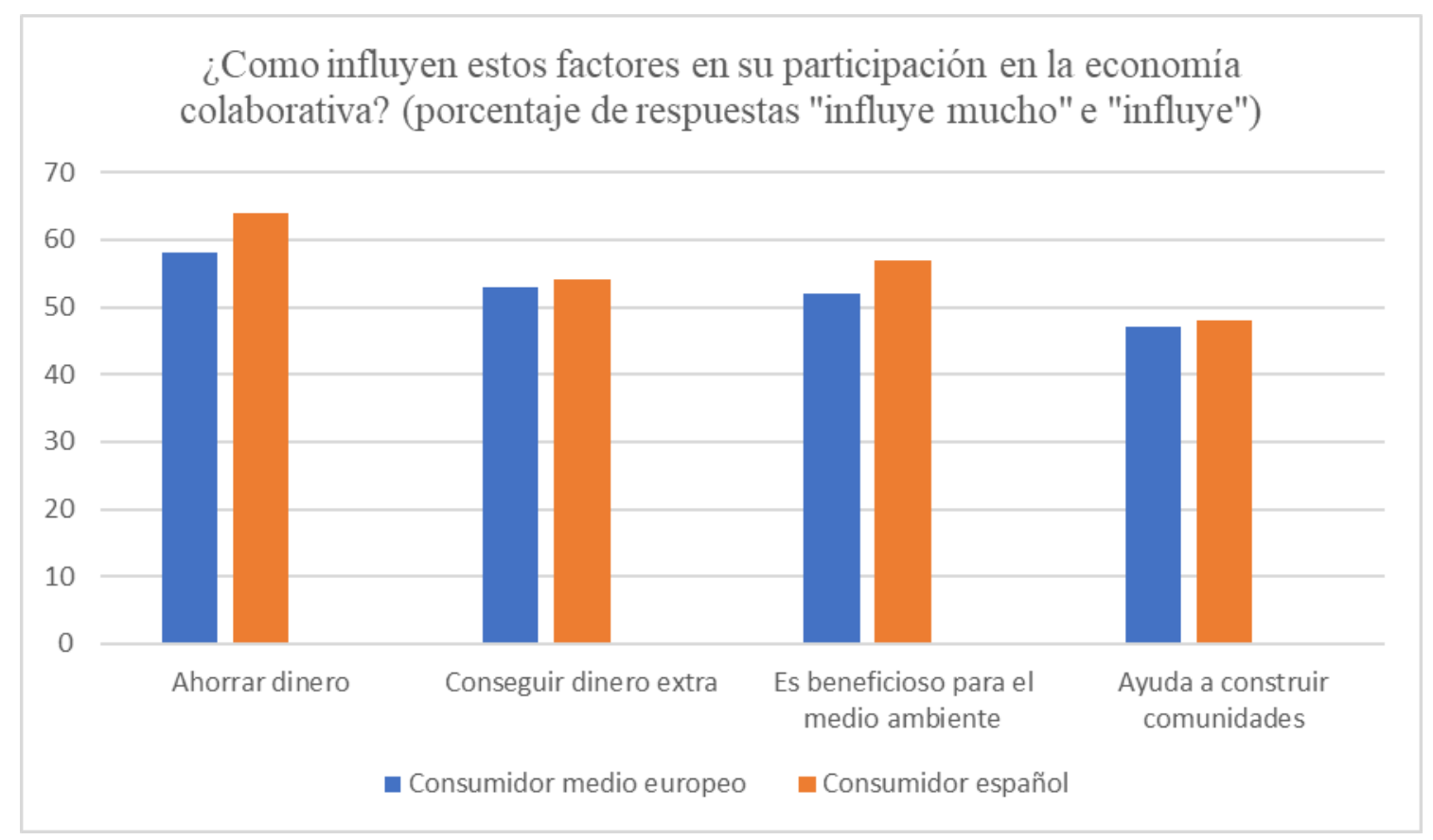

Como puede apreciarse en las respuestas, la oportunidad de ahorrar dinero es el factor más influyente a la hora de utilizar estas plataformas, lo cual es un dato común en los 15 países donde se realizó la encuesta, abarcando no solo Europa sino también Australia y Estados Unidos. En segundo lugar aparece la oportunidad de ganar un dinero extra y por detrás la sensibilidad medioambiental; con la excepción de España, donde estos factores aparecen en orden inverso. Resulta relevante el último lugar que ocupa el factor "ayuda a construir comunidades", lo que en nuestro criterio indica que la economía colaborativa es percibida en primer lugar como una oportunidad económica por la reducción de costes o la facilidad para acceder a un mercado global. En segundo lugar, es también asociada a un consumo eco-responsable, pero en menor medida como un modelo socialmente responsable.

Un dato curioso es que el sentido del factor "ayuda a construir comunidades" se refería a la posibilidad de ampliar las relaciones entre ofertantes y usuarios, por ejemplo, a través de la creación de tiendas comunitarias compartidas ("community shops"). Estas son en esencia tiendas en zonas rurales, en régimen de propiedad común o compartida de forma democrática por los miembros de la comunidad. Funcionan con un criterio empresarial pero su finalidad es el beneficio de la comunidad. Se rigen por el principio de adhesión voluntaria, por lo que cualquier persona que resida en la localidad puede comprar los bienes a la venta en estas tiendas, pero también participar de la gestión del negocio como propietario comunero, según el principio de una persona un voto, es decir, con igual cuota de decisión sin tener en consideración su aportación o inversión. En cuanto a la gestión, existen varios modelos; la mayoría de estas tiendas comunitarias son gestionadas por la propia comunidad, en ocasiones con la ayuda de voluntarios, pero también contratando asalariados. Estas experiencias han resultado notablemente exitosas en el Reino Unido, donde según datos estadísticos han resultado una forma muy resiliente de negocios: de las cerca de 350 tiendas comunitarias abiertas en Inglaterra, Escocia y Gales en los últimos años, solo 15 han tenido que cerrar, lo que arroja un $95 \%$ de supervivencia, un dato extremadamente positivo en comparación con la 
estimación de un $41 \%$ como ratio de supervivencia empresarial en los primeros cinco años de los pequeños negocios en el Reino Unido (Plunkett Foundation, 2017).

También es relevante que la percepción de que la economía colaborativa ayuda a crear comunidades fuera un factor influyente para el $73 \%$ de los que ya participaban en alguna experiencia colaborativa ("sharers"), mientras que solo el 39\% de quienes no participaban en estas experiencias ("non-sharers") consideraron que este fuera un factor influyente para decidir utilizar estas plataformas. Esto revela una mayor sensibilidad hacia la responsabilidad social entre quienes "están dentro" o forman parte de alguna red colaborativa, en comparación con quienes solo utilizan ocasionalmente las plataformas o se plantean utilizarlas. También pudo establecerse un cierto perfil de los participantes o sharers, al definirse en su mayoría como consumidores cualificados y por debajo de los 35 años.

En cuanto a la sensibilidad medioambiental, el género marca una cierta diferencia, pues el $57 \%$ de las mujeres encuestadas declaran que una importante motivación para al uso de estas plataformas es la protección del medio ambiente, frente a un 50\% de los hombres encuestados. De forma similar, es mayor la proporción de mujeres que piensan en la economía colaborativa como una forma de ahorro, $61 \%$, frente a un $56 \%$ de los hombres.

Una apreciación a nuestro juicio relevante es que estos cuatro factores son, al mismo tiempo, auténticas motivaciones de compra. Por tanto, podrían ser utilizados en campañas de marketing por grandes empresas, aprovechándose, por ejemplo, de la especial sensibilidad medioambiental en los últimos tiempos; para confundirse con las auténticas organizaciones de economía social o colaborativa, con la principal o única finalidad de incrementar sus ingresos. De ahí que se ponga en duda por muchos autores la apariencia de economía colaborativa de algunos de estos modelos, tras los que muchas veces se esconden empresas o plataformas claramente capitalistas.

\section{Cooperativismo o capitalismo de plataforma}

La dificultad en definir los distintos modelos que van surgiendo y desarrollándose impulsados por las nuevas tecnologías y alrededor de las plataformas digitales, así como precisar si realmente se enmarcan en la economía social o colaborativa, aconseja una conceptualización que refleje de forma dinámica la idea de evolución o continuo.

Para Cañigueral (2016), es importante reconocer la gran diversidad de negocios colaborativos y poderlos ubicar en algún punto entre las plataformas de corte totalmente capitalista y las plataformas de corte cooperativista. Para ello propone el Sharing Business Model Compass, un modelo creado por Boyd Cohen y Pablo Muñoz identificando un buen número de dimensiones consideradas clave de las startups de la economía colaborativa. Este modelo permite organizar las iniciativas de manera coherente y sistematizada, pero con una perspectiva dinámica, lo que facilita el cambio o evolución de un modelo a otro.

El Sharing Business Model Compass establece seis dimensiones distintas para los modelos de economía colaborativa: tecnología, transacción, enfoque de negocio, recursos compartidos, modelo de gobernanza y tipo de plataforma. Cada dimensión tiene tres grados, que representan un continuo desde, por ejemplo, los modelos de gobierno con propiedad y control concentrados, hasta una propiedad y control completamente participativo. Cuatro de las dimensiones (tipo de plataforma, gobernanza, modelo de negocio y transacción) van desde las más comúnmente encontradas en las startups de economía colaborativa, basadas en el mercado, a modelos cooperativos más centrados en la idea del bien común. Las dimensiones tecnología y recursos compartidos no son un continuo en la misma forma, sino que los modelos de negocio de mercado o del bien común pueden utilizar cualquiera de las tres opciones ofrecidas. 
Gráfico. 3. Sharing Business Model Compass. Fuente: Cohen y Muñoz (2016, en Cañigueral, 2016).

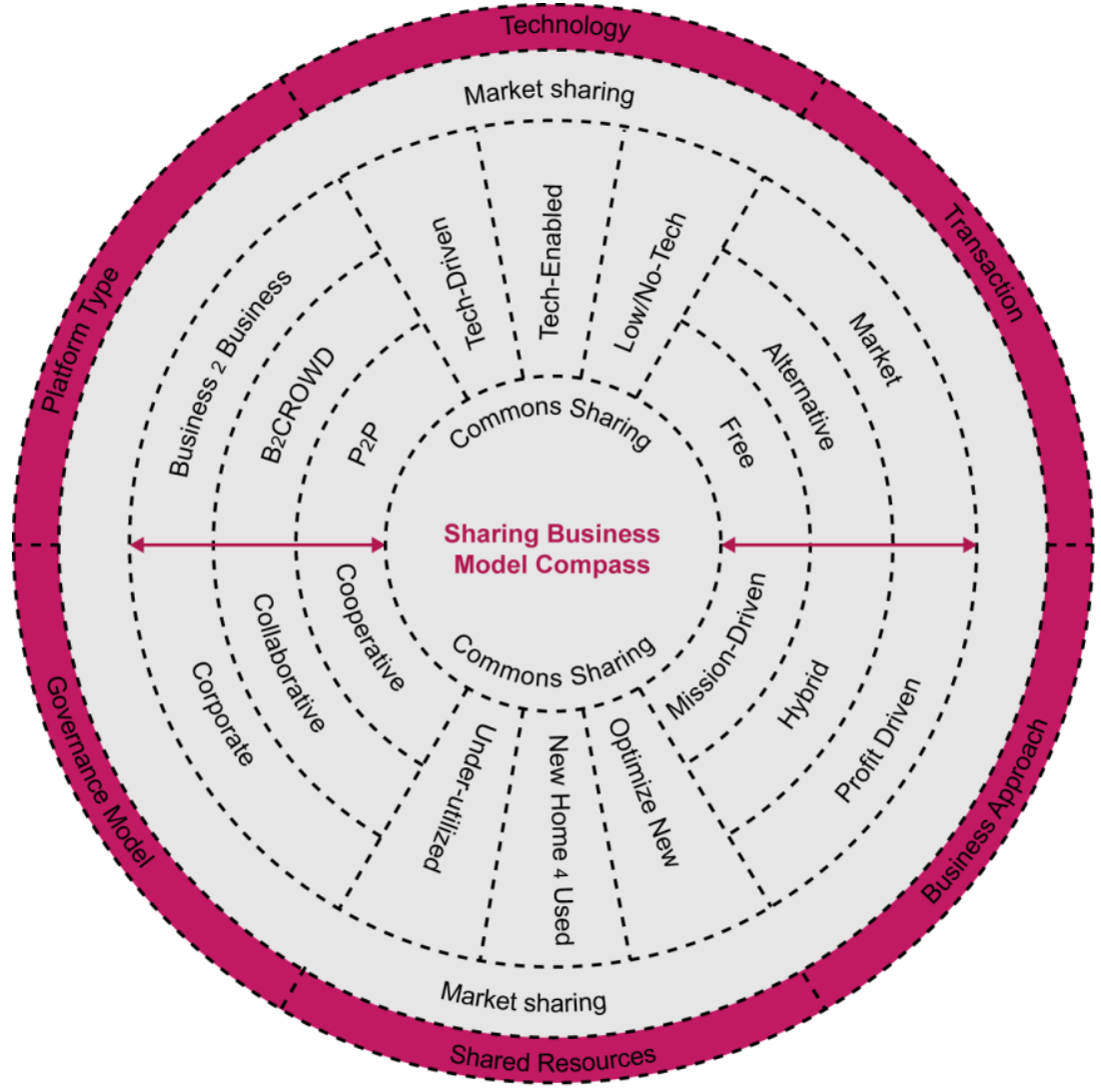

Como anteriormente se ha referido, la economía colaborativa engloba a los nuevos sistemas de producción y consumo de bienes y servicios surgidos que se intercambian y comparten a través de plataformas digitales. Sin embargo, muchos estudios apuntan que la economía colaborativa tiene poco de este adjetivo pues tales plataformas no comparten ni la propiedad ni los beneficios con los usuarios que son quienes generan valor dentro de ellas (Alfonso, 2106).

En su obra Cooperativismo de plataforma: desafiando la economía colaborativa corporativa, Trebor Scholz (2016), concluye que la economía colaborativa corporativa "no es simplemente una continuación del capitalismo predigital tal como lo conocemos, hay notables discontinuidades, un nuevo nivel de explotación y una mayor concentración de la riqueza". Y añade que "las infraestructuras digitales están diseñadas para extraer provecho para un número muy reducido de propietarios de plataformas y accionistas".

El modelo de cooperativismo de plataforma que Scholz propone surge como un movimiento en el que las plataformas pasan a ser propiedad de quienes proporcionan los recursos que las hacen funcionar, cualquiera que sea su papel en ellas, es decir, tanto si aportan trabajo, bienes o actúan como consumidores. Otro factor clave es el referido a su gobernanza, que debe ser democrática y expresarse en un reparto de beneficios entre sus copropietarios productores-consumidores. Es, en síntesis, la extensión de los fundamentos de las cooperativas a las plataformas colaborativas; una manera diferente de hacer economía colaborativa (Alfonso, 2016).

Dentro de los considerados valores cooperativos puede hablarse de valores básicos, tales como ayuda mutua, autorresponsabilidad, democracia, igualdad, equidad y solidaridad; y valores éticos tales como honestidad, transparencia, responsabilidad social y preocupación por los demás. De ellos deriva que es responsabilidad de la cooperativa no sólo la obtención de un beneficio económico sino también la mejora de la calidad de vida y de la situación económica de los socios, así como de la comunidad dónde desarrolla su actividad (Jaén, 2017).

Los principios y valores cooperativos suponen además una ventaja clave para estas entidades, que es su capacidad para proporcionar acceso a la información y vincular sus miembros a los mercados mientras que ellos, actuando individualmente, normalmente no tendrían ese acceso. Marcuello y Saz (2008, en García, Román y García Martínez, 2016) concluyen en que el cumplimiento de los principios cooperativos posibilita la generación de capital social en las cooperativas, lo que influye en la capacidad de absorción de conocimiento por parte de la organización y, por tanto, en la innovación.

Para Vicente, Parra y Flores (2017), puede hablarse de una relación de paralelismo real entre la economía social, las sociedades cooperativas en particular y la economía colaborativa, siendo internet, y en especial las 
plataformas digitales los elementos motores del cambio y evolución de algunas formas de la primera a ciertas prácticas de la segunda. Dicha afinidad se aprecia, por ejemplo, cuando en ambos casos existe una variedad terminológica para referir realidades semejantes y por la consideración de las personas como el elemento vertebrador de ambos modelos, junto a sistemas de funcionamiento paritario.

Estos puntos de conexión permitirían que ambos modelos desarrollaran en mayor medida las potencialidades que tienen: la economía social ampliar el alcance de las actividades que realizan las organizaciones que la forman; la economía colaborativa trasladar al mundo offline los resultados que genera y reforzar el sentido de comunidad a través de la actividad que realizan sus agentes.

Ambos modelos muestran una tradición diferente: por un lado, la economía social se nutre del asociacionismo histórico que surgió con la revolución industrial; por otro, la economía colaborativa emerge como ecosistema transformador, introduciendo modelos de negocio novedosos, a partir del desarrollo de internet. Aunque no resultan asimilables, algunas corrientes han intentado combinarlos y se habla de "economía social colaborativa" o "economía colaborativa social", para poner de relieve sus posibles coincidencias (Díaz, Marcuello y Monreal, 2016).

En un sentido opuesto, otros autores centran su análisis en la desviación de algunas de las cooperativas de mayor peso en el mercado hacia prácticas competitivas, más propias de modelos corporativos, en perjuicio de los valores cooperativos, y proponen la reformulación de ciertos conceptos pero haciendo converger tal revisión con la nueva realidad de la economía colaborativa. Surge en este caso el llamado "open cooperativism", como derivado del cooperativismo tradicional en confluencia con la economía colaborativa (Alfonso, 2016).

No es extraño entonces que Scholz (2016) introdujera el término "cooperativismo de plataforma" (Platform Coop), para diferenciar este modelo del "capitalismo de plataforma" y como expresión de una economía colaborativa que combina lo mejor de las plataformas digitales con los principios cooperativistas internacionales. Esto es, con la idea de propiedad compartida, la puesta en marcha de nuevos modelos de gobernanza, la participación del ciudadano productor de valor en la toma de decisiones en términos de igualdad y el reparto de los beneficios entre los usuarios. De esta manera, el valor añadido del cooperativismo de plataforma es generado por todos los agentes que interactúan a través de la plataforma digital (Alfonso, 2016). Este concepto tiene importantes coincidencias con el de "innovación social", que se define como nuevas ideas, modelos y servicios que tienen la virtud de satisfacer nuevas necesidades sociales y de bienestar desde nuevas relaciones entre los agentes implicados desde una cultura colaborativa (Murray, Caulier-Grice y Mulgan, 2010).

Las diferencias entre los modelos de cooperativismo y capitalismo de plataforma han sido expuestas al comparar la propuesta de Fairbnb frente al modelo de negocio de Airbnb (Enciso Santocildes, M., Mugarra Elorriaga, A., Muñecas Izaguirre, I. y Ruíz Martínez, 2019). El primer caso se basa en la tradición europea de la economía social y solidaria y sus principios inspiradores se encuentran en el marco de la economía de cooperación; se busca así innovar de forma responsable, participativa, comunitaria y sostenible.

Según exponen los creadores de esta iniciativa, (Fairbnb.coop Manifesto, 2019) "las plataformas de alquiler vacacional convencionales tienen tres problemas principales: son opacas, generan grandes costes en la comunidad local y son antidemocráticas. (...) De Ciudad del Cabo a Tokio, pasando por Nueva York o Barcelona, los barrios han experimentado un aumento de los precios del mercado inmobiliario, la erosión de las comunidades y el desplazamiento de muchas familias. Las plataformas vacacionales surgieron para facilitar el alquiler de alojamientos vacacionales entre particulares. Sin embargo, bajo la bandera de permitir a los residentes pertenecer a cualquier parte, estas plataformas han facilitado un modelo irresponsable de turismo en el que en muchas ocasiones el alquiler no es entre particulares y que en muchos casos sucede a espaldas de la legalidad.

Para muchos ciudadanos, la promesa de "compartir la casa" ha facilitado que su familia sea despojada de la suya. Asociaciones de vecinos, movimientos sociales y gobiernos locales han expresado su creciente preocupación ante la gentrificación asociada con el turismo y el deterioro de las condiciones de vida que esto conlleva. Incluso la industria hotelera ha empezado a reconocer el problema del exceso de turismo.".

Para dar solución a estos problemas se crea el modelo Fairbnb.coop, dando prioridad a la transparencia, la distribución de beneficios a las comunidades y la toma de decisiones de forma democrática. En la práctica esto se traduce, entre otras medidas, por la colaboración con los municipios para garantizar la legalidad de todas las viviendas alquiladas y el pleno cumplimiento de la legislación local. Además, la mitad de la comisión cobrada por Fairbnb.coop se devuelve a la comunidad local, sosteniendo proyectos seleccionados por los residentes locales, que pueden ser zonas de juego, centros culturales, jardines comunitarios o refugios para mujeres. De esta forma los ingresos de los viajeros alimentan y regeneran las comunidades.

En cuanto a la forma jurídica, Fairbnb se constituye como una cooperativa, propiedad de sus miembros. Así se garantiza su independencia, porque la plataforma no es propiedad de inversores desconocidos sino de aquellos que trabajan en ella, de quienes la usan y quienes sienten el impacto de su uso. No es solo un modelo de copropiedad, sino también de co-gobernanza. Las decisiones se toman de forma colaborativa a través de un modelo de gobernanza distribuida. Se establecen como criterios para su funcionamiento valores 
y políticas éticas, que van más allá del criterio empresarial, lo que se manifiesta, por ejemplo, en la limitación de los salarios de sus miembros. Esto permite, a través de su red de nodos locales, empoderar a las comunidades locales para que den forma al turismo que les afecta, y asegurar que sus beneficios vuelvan a ellos. Estos nodos locales están en el centro de la organización y representan el motor que conecta a las personas en los territorios haciendo de la interacción humana la verdadera "tecnología" de Fairbnb.coop.

En esta propuesta tiene gran importancia la transparencia y el acceso a la información, por lo que se comparten datos no personales sobre sus transacciones con administraciones públicas de forma que puedan entender el impacto real del turismo en su área. Igualmente, se aplica la política ' 1 usuario - 1 casa' para prevenir la existencia de 'multi-hosts' en la plataforma, limitando así el efecto de los alojamientos vacacionales en el mercado de vivienda.

Sus propios creadores se definen como una comunidad de activistas, programadores, investigadores y diseñadoras que aspiran a solucionar el reto de poner el procomún en el centro de la economía colaborativa.

Otra iniciativa similar es el modelo de economía colaborativa procomún, desarrollado por el grupo de trabajo Barcelona Colaborativa desde el 2015 (Cañigueral 2016), aunque fue en marzo de 2016, durante el encuentro Procomuns, que se presenta la "Declaración y propuestas de nuevas políticas para la economía colaborativa procomún". En ella se describen, de forma general y sin establecer características excluyentes, los rasgos de este nuevo modelo, entre los que pueden citarse:

Producción colaborativa en torno a una plataforma colaborativa que no se limita a la intermediación, conectando oferta y demanda o proveedores con usuarios; aquí la propia plataforma es una herramienta de producción que favorece la cocreación interactiva.

Relaciones P2P o entre iguales, fomentando esta cocreación en un contexto de comunidad de usuarios, más allá del marco contractual o mercantil.

Recursos comunes: el proceso genera colectivamente bienes que se pueden disfrutar, adaptar o replicar de forma abierta. Esto supone tanto el acceso libre de los usuarios al servicio y a la información generada, para el uso, intercambio, consumo o producción entre ellos como la apropiación colectiva: ofrecen o generan recursos compartidos con licencias claras de reutilización, ya sea para permitir obras derivadas, o una reutilización que genere nuevas oportunidades económicas y capital social (sin dañar el bien compartido).

También en Barcelona, durante el OuiShare Fest de 2017 (Portal de Economía Solidaria, 2017), se avanzó en una configuración de lo que han llamado "Economía Social y Solidaria", un modelo con la capacidad de usar los valores para transformar hábitos de consumo. En él las personas ocupan un lugar central pero no solamente como objetivo de la actividad económica, destinada a la satisfacción de sus necesidades, sino como productores y creadores de valor, porque el empoderamiento de las personas mediante el uso de herramientas tecnológicas y la cooperación es clave tanto para la economía social y solidaria como para la colaborativa.

Precisamente el uso de esas herramientas tecnológicas se revela un factor clave en el rumbo que pueda seguir la evolución de estos modelos. En palabras Klaus Schwab, creador del Foro de Davos y autor del libro "La cuarta revolución industrial", estamos al borde de una revolución tecnológica que modificará fundamentalmente la forma en que vivimos, trabajamos y nos relacionamos. En su escala, alcance y complejidad, la transformación será distinta a cualquier cosa que el género humano haya experimentado antes. Esta llamada cuarta revolución industrial está marcada por la convergencia de tecnologías digitales, físicas y biológicas; y no se trata del desarrollo de estas tecnologías por separado, sino del encuentro de esos desarrollos, su convergencia e integración. El principio básico es que las empresas podrán crear redes inteligentes que se controlarán a sí mismas, a lo largo de toda la cadena de valor.

Un ejemplo de esta capacidad de autocontrol es la tecnología blockchain que, definida en una frase, (Prados Rodríguez, 2018:196) "sirve para tener la confianza de que una transacción se ha producido sin necesidad de una autoridad central, es un sistema o protocolo para la descentralización de la confianza".

Precisamente la confianza está en el centro de las diferentes actividades de la economía colaborativa. Originalmente esta se establecía entre personas cercanas, para luego extenderse por las redes mediante los diferentes sistemas de reputación. Además, "la economía colaborativa verdadera se basa en una comunidad de iguales que colabora, coopera y comparte en red mediante sistemas justos que buscan el bien de la comunidad en que se insertan. Estos son los fundamentos en que se basan las actividades de economía colaborativa entendidas conforme a su naturaleza: la cooperación entre iguales para un bien común" (Gómez-Álvarez y Morales, 2018, citados por Prados Rodríguez, 2018:209). Por tanto, la tecnología blockchain ofrece una oportunidad de darle autenticidad a la colaboración a través de la descentralización que promueve, lo que permitiría una reducción de los costes de intermediación, facilitar la gobernanza, incluyendo sus mecanismos de control, mediante sanciones e incentivos a los comportamientos que se quieren penalizar o fomentar; así como incrementar la transparencia y favorecer la soberanía económica, incluyendo la emisión de moneda.

De esta forma, se podría erigir como facilitador de los tres pilares de la economía colaborativa verdadera. En primer lugar, "favorece el uso de los recursos y las interacciones sociales, porque el blockchain es un registro inmutable de nuestra reputación que se crea por la interacción con otros participantes, y esta es 
pública, frente al sistema actual donde la reputación creada en una plataforma no se puede trasladar a otra. De hecho, el papel actual de las plataformas capitalistas es hacer de "guardianes" de la confianza creada por la interacción de muchos participantes. En segundo lugar, el blockchain facilita la interacción directa entre peer-to- peer, por tanto reduce el poder de las plataformas. (...) Por último, el blockchain permite que el valor del ecosistema no sea acumulado en una compañía o marca central, sino que el valor final generado del mismo sea distribuido entre los participantes que han aportado un valor inicial a la comunidad". (Prados Rodríguez, 2018:208)

Sin embargo, aunque los principios de descentralización que impulsaron la creación del protocolo de cadena de bloques están compartidos con la economía colaborativa, también pueden actuar en sentido contrario a los fines que esta persigue, por ejemplo, favoreciendo economías de escala que impulsan una economía de competición en lugar de colaboración, centralizando los procesos de toma de decisiones en los operadores especializados (como los mineros en el caso de bitcoin), estableciendo una falsa transparencia o propiciando que la distribución del beneficio del uso de la red sea asimétrica. Por todo ello coincidimos en que "debemos centrar nuestros esfuerzos para que la tecnología como herramienta trabaje para el bien común, fomentando de forma correcta la economía colaborativa, evitando los riesgos de considerar el código como ley para en su lugar embeber leyes más humanas en el código" (Prados Rodríguez, 2018:211).

\section{Conclusiones}

Desde un punto de vista técnico doctrinal resulta difícil definir o delimitar los distintos modelos que van surgiendo y desarrollándose impulsados por las nuevas tecnologías y alrededor de las plataformas digitales. Cualquier concepto o definición al respecto es un intento de fijar y acotar un fenómeno en crecimiento, de ahí su complejidad, pero a la vez es esta diversidad es expresión de su potencial y riqueza. En algunos casos se ha enfatizado en los paralelismos que pueden apreciarse entre la economía colaborativa y la economía social, pero un análisis más profundo pone de relieve que estos modelos no discurren en paralelo, más bien se conectan e influyen mutuamente, hasta el punto en que una organización o un proyecto puede tener su origen en una forma de economía colaborativa y evolucionar hacia formas de economía social, y viceversa. En consecuencia, la conceptualización más precisa podría ser aquella que refleje de forma dinámica la idea de evolución o continuo.

Desde esta perspectiva dinámica, la economía colaborativa se situaría en un espacio intermedio, pues en ocasiones tiene más semejanzas con la economía tradicional, de la que pretende ser un modelo alternativo, cuando las plataformas "colaborativas" solo están diseñadas para reducir costes y maximizar beneficios. Este modelo del "capitalismo de plataforma", aunque formalmente similar, resulta en esencia opuesto al "cooperativismo de plataforma", un modelo de economía colaborativa que combina lo mejor de las plataformas digitales con los principios cooperativistas internacionales. El cambio de uno a otro es consecuencia de la idea de propiedad compartida, la puesta en marcha de nuevos modelos de gobernanza, la participación del ciudadano productor de valor en la toma de decisiones en términos de igualdad y el reparto de los beneficios entre los usuarios.

Precisamente las nuevas tecnologías puedes favorecer o hacer posible nuevos modelos de propiedad en común caracterizados por la gobernanza colaborativa. Sin embargo, es obvio que la cuarta revolución industrial por sí sola no va a alumbrar una nueva era de equidad y bienestar. Las plataformas digitales, y en general la tecnología como herramienta, puede ser usada para maximizar beneficios, muchas veces a costa del medio ambiente y del deterioro del entorno local; pero también puede ponerse la innovación al servicio de las personas y la sociedad. En ambos casos depende de los valores y principios que sirvan de fundamento al uso de dichas plataformas. No se trata de proclamar formalmente tales principios, porque como hemos visto las supuestas entidades de economía social se han visto arrastradas por la crisis en la misma medida que las empresas capitalistas. La verdadera esencia de este modelo social y colaborativo debe traducirse efectivamente en su funcionamiento y gestión, es decir, en la gobernanza democrática y colaborativa.

En este sentido, los valores cooperativos, tal y como han evolucionado con el paso del tiempo, pueden servir como guía y adaptarse a estas nuevas actividades económicas que orbitan alrededor de las plataformas digitales e impulsar la transformación de las organizaciones desde el modelo corporativo al cooperativo, pasando por el colaborativo. Esto significaría no solo una profunda transformación a nivel económico, que deberá ir acompañada de una regulación y un marco jurídico adecuado, sino también una transformación social, un impacto cultural y un nuevo paradigma más sostenible y humanista, centrado en el libre acceso a los bienes y servicios comunes, la reutilización de recursos compartidos y la cocreación cooperativa.

Precisamente, el reto actual es poner el procomún en el centro de la economía colaborativa y la cuarta revolución industrial puede ser una oportunidad para ello porque la tecnología ya lo permite. Ahora solo falta garantiza el acceso democrático a esa tecnología y utilizarla de forma social y eco-responsable para garantizar modelos de negocio centrados en las personas y que impulsen la innovación social con una perspectiva participativa y humanista. 


\section{Referencias bibliográficas}

Alfonso Sánchez, R. (2016) Economía colaborativa: un nuevo mercado para la economía social, CIRIEC-España, Revista de Economía Pública, Social y Cooperativa, No 88, pp. 231-258.

Botsman, R. y Rogers, R. (2010) What's Mine is Yours: The Rise of Collaborative Consumption. Edición internacional. Collins Business.

Cañigueral, A. (2016) Hacia una economía colaborativa «responsable». OIKONOMICS, Revista de los Estudios de Economía y Empresa, $\mathrm{N}^{\circ}$ 6, pp. 16-27.

Díaz Foncea, M., Marcuello, C., y Monreal, M. (2016) Economía social y economía colaborativa: encaje y potencialidades. Revista de Economía Industrial, No 402, pp. 27-35.

Enciso Santocildes, M., Mugarra Elorriaga, A., Muñecas Izaguirre, I. y Ruíz Martínez, A. (2019) Economía colaborativa vs economía de plataforma: el caso Fairbnb y sus principios inspiradores desde la economía de cooperación. Boletín de Estudios Económicos, Vol. LXXIV, № 227, pp. 259-283.

Fairbnb.coop Manifesto (2019) Disponible en: https://social.fairbnb.coop/posts/fairbnbcoop-manifesto.

Fajardo García, G. (2018) La identificación de las empresas de economía social en España. Problemática jurídica. REVESCO. Revista de Estudios Cooperativos, Segundo Cuatrimestre, $\mathrm{N}^{\mathrm{o}}$ 128, pp. 99-126. DOI: 10.5209/REVE.60209.

Gansky, L. (2010) The mesh: why the future of business is sharing. Nueva York: Penguin.

García Pérez, A. M., Román Cervantes, C. y García Martínez, M. (2016) De la cooperativa agroalimentaria a la "learning netchain". Hacia un planteamiento teórico interorganizativo e interpersonal. REVESCO Revista de Estudios Cooperativos, Segundo Cuatrimestre, No 121, pp.114-144. DOI: 10.5209/rev_REVE.2016.v121.51304.

García, M. J. (2017) Crisis económica y economía social. REVESCO. Revista de Estudios Cooperativos, Monográfico, No 126, pp. 74-93. DOI: 10.5209/REVE.58394.

García-Gutiérrez Fernández, C (1988) El problema de la doble condición de los socios-trabajadores (socios-proveedores y socios-consumidores) ante la gerencia de la empresa cooperativa. REVESCO. Revista de Estudios Cooperativos, $\mathrm{N}^{\mathrm{0}} 56, \mathrm{pp} .83-122$.

ING International (2015) The European sharing economy set to grow by a third in the next 12 months. Disponible en http://www.ing.com/Newsroom/All-news/European-sharing-economy-to-grow-by-a-third-in-the-next-12months.htm.

Iturrioz del Campo, J. (2001) Las sociedades de garantía recíproca como empresas de participación. CIRIEC-España, Revista de Economía Pública, Social y Cooperativa, No 38, pp. 133-154.

Jaén García, M. (2017) Crisis económica y economía social. REVESCO. Revista de Estudios Cooperativos, Monográfico, $\mathrm{N}^{\circ} 126$, pp. 74-93. DOI: 10.5209/REVE.58394.

Mariñez Navarro, F. (2016) Innovación y gobernanza colaborativa para la gestión de las políticas públicas. XXI Congreso Internacional del CLAD sobre la Reforma del Estado y de la Administración Pública, Santiago, Chile, 8 11 nov. 2016. Disponible en https://www.researchgate.net/publication/324314487.

Murray, R., Caulier-Grice, J. y Mulgan, G. (2010) The Open Book of Social Innovation, Londres, The Young Foundation \& Nesta.

OPCIONES (2013) Economías colaborativas. Revista Opciones, N 44. Disponible en http://opcions.org/es/revista/44economias-colaborativas.

Ostelea, Escuela de Management en Turismo y Centro de Investigación, Divulgación e Innovación en Turismo (IDITUR) (2018) Plataformas de economía colaborativa: una mirada global. Disponible en: http://www.aept.org/archivos/documentos/ostelea_informe_economia_colaborativa.pdf.

OUISHARE (2016) Definición de economía colaborativa. OUISHARE, connecting the collaborative economy. Disponible en: http://ouishare.net/es/.

Plunkett Foundation (2017) Community Shops. A better form of business 2017. Disponible en: http://www.farmboroughshop.co.uk/wp-content/uploads/2018/03/Plunkett_BetterBusiness_Shops_final-2017.pdf.

Portal de Economía Solidaria (2017) 5 Puntos de Encuentro entre la Economía Colaborativa y la Economía Social y Solidaria. Disponible en https://www.economiasolidaria.org/biblioteca/5-puntos-de-encuentro-entre-la-economiacolaborativa-y-la-economia-social-y-solidaria.

Prados Rodríguez, M. (2018) Introducción a la tecnología blockchain (cadena de bloques). En: Gómez-Álvarez Díaz, R., Patiño Rodríguez, D. y Plaza Angulo, J. J. (directores) Economía colaborativa... ¿de verdad? $1^{\mathrm{a}}$ edición, Murcia, Ediciones Laborum S.L.

Sajardo Moreno, A. y Pérez Sempere, S. (2019) Análisis de las plataformas de crowdfunding social en el estado español: un estudio de casos. REVESCO. Revista de Estudios Cooperativos, Primer Cuatrimestre, № 130, pp. 149175. DOI: 10.5209/REVE.61938.

Scholz, T. (2016) Cooperativismo de plataforma. Desafiando la economía colaborativa corporativa. Barcelona: Publicaciones Dimmons, Disponible en: http://dimmons.net/wp-content/uploads/2016/05/maq_TreborScholz_COOP_PreF_DP.pdf.

SHES (2016) Definición de economía colaborativa. Asociación Sharing-España: Disponible en: http://www.sharingespana.es/quienes-somos/.

SHES (2017) Los modelos colaborativos y bajo demanda en plataformas digitales. Disponible en: https://www.adigital.org/informes-estudios/los-modelos-colaborativos-demanda-plataformas-digitales/.

Tapscott, D. y Williams, A. D. (2010) Wikinomics. Nueva York: Penguin. 
Vaughan, R. y Daverio, R., PwC UK (2016) Assessing the size and presence of the collaborative economy in Europe. Disponible en www.pwc.co.uk.

Vicente, A., Parra, M. C. y Flores, M. P. (2017) ¿Es la Economía Colaborativa una versión 2.0 de la Economía Social? Sphera Publica, No 1, Vol. 17, pp. 64-80. 